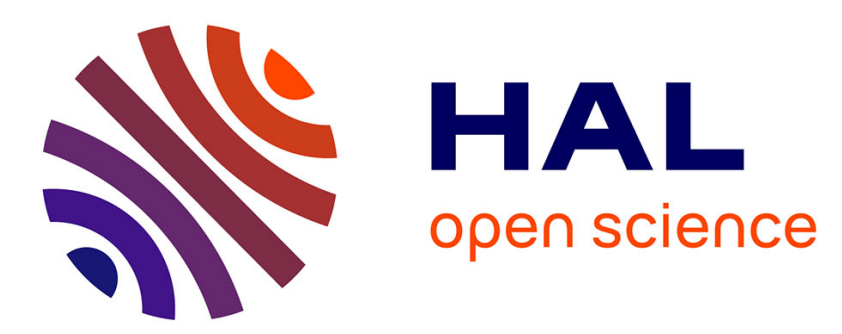

\title{
Study of Reconfigurable Suspended Cable-Driven Parallel Robots for Airplane Maintenance
}

\author{
Dinh Quan Nguyen, Marc Gouttefarde
}

\section{To cite this version:}

Dinh Quan Nguyen, Marc Gouttefarde. Study of Reconfigurable Suspended Cable-Driven Parallel Robots for Airplane Maintenance. IROS: Intelligent RObots and Systems, Sep 2014, Chicago, IL, United States. pp.1682-1689, 10.1109/IROS.2014.6942781 • lirmm-01221408

\section{HAL Id: lirmm-01221408 https://hal-lirmm.ccsd.cnrs.fr/lirmm-01221408}

Submitted on 1 Dec 2018

HAL is a multi-disciplinary open access archive for the deposit and dissemination of scientific research documents, whether they are published or not. The documents may come from teaching and research institutions in France or abroad, or from public or private research centers.
L'archive ouverte pluridisciplinaire HAL, est destinée au dépôt et à la diffusion de documents scientifiques de niveau recherche, publiés ou non, émanant des établissements d'enseignement et de recherche français ou étrangers, des laboratoires publics ou privés. 


\title{
Study of Reconfigurable Suspended Cable-Driven Parallel Robots for Airplane Maintenance
}

\author{
Dinh Quan Nguyen, Marc Gouttefarde*
}

\begin{abstract}
This paper discusses the use of large-dimension reconfigurable suspended cable-driven parallel robots (CDPR) to substitute for conventional gantry nacelles that carry workers in an airplane maintenance workshop. The reconfiguration of the CDPR is considered as a multi-objective optimization problem with two performance indices. One criterion is the sum of the cable tensions which is directly related to the power consumption of the CDPR. The other criterion is the normalized upper bound on the infinitesimal displacement of the mobile platform, used here as a means to quantify the stiffness of the CDPR. A systematic procedure is proposed to find optimal reconfigurations of the CDPR while dealing efficiently with the tradeoff between these two criteria.
\end{abstract}

\section{INTRODUCTION}

Cable-driven parallel robots have been a subject for research in the past twenty years. Because of their appealing advantages such as light weight, large workspace, high load capacity, ease of construction, ease of reconfiguration and low cost, many studies have been carried out on the use of CDPR to replace conventional methods of handling heavy payloads across wide workspaces [1]-[12].

Let us consider an example in the aerospace industry. Fig. 1 shows the airplane maintenance operations ${ }^{1}$ in which, several gantry nacelles are used to carry the workers across the airplane fuselage to perform certain maintenance tasks. Each telescopic platform can weigh up to 11 tons which implies high costs for the building construction. Moreover,

* Dinh Quan Nguyen and Marc Gouttefarde are with the Laboratoire d'Informatique, de Robotique et de Micro-électronique de Montpellier (LIRMM-CNRS-UM2), 161 rue Ada, 34392 Montpellier Cedex 5, France dinhquan.nguyen@lirmm.fr, marc.gouttefardedirmm. fr

${ }^{1}$ https://blog.klm.com/not-just-any-paint-job/1533/

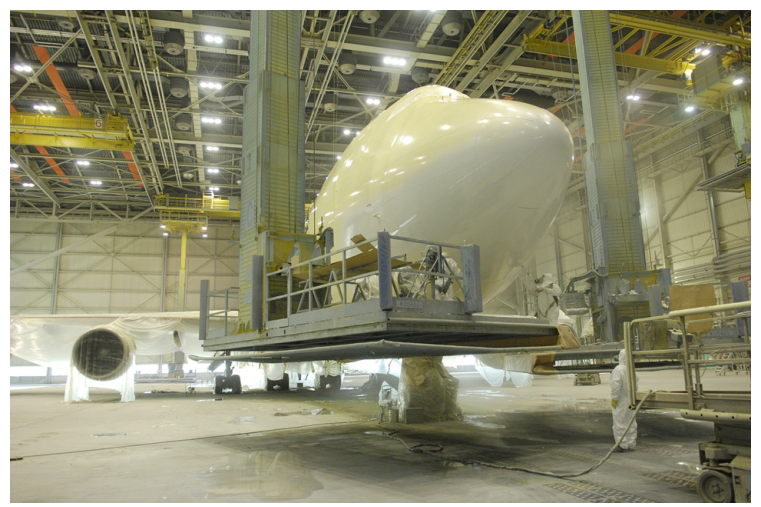

Fig. 1: Carrying workers by gantry nacelles in an airplane maintenance workshop large power sources may be needed to drive such heavy systems. By using large-dimension reconfigurable CDPR to replace the gantry nacelles, one could reduce the cost of construction.

Recent studies [12]-[14] deal with new types of CDPR which can be reconfigured by changing their cable layout. Reconfigurability offers more flexible choices to the endusers and should improve the CDPR performances. Several systematic procedures have been proposed to determine the design solution for reconfigurable CDPR e.g. in [13], [14]. These approaches aim to find optimal reconfigurations for the CDPR by optimizing a certain performance index. Possible criteria are the dexterity of the CDPR [13] or the tension factor (which is the ratio between minimal and maximal values of cable tensions along a given trajectory) [14]. However, these two previous studies only dealt with planar CDPR. For a general 6-DOF CDPR, the problem becomes much more complicated notably since one needs to take into account additional constraints such as cable interferences.

In a previous work [15], as illustrated in Fig. 2, we studied a reconfigurable CDPR that could be used to replace the conventional method to handle heavy payloads across a wide workshop. This CDPR is highly redundant with 14 actuators. The mobile platform is driven by 8 cables. The general suspended redundant architecture of the CDPR is kept similar to that of the CoGiRo prototype [11] in order to obtain a large Cartesian workspace as well as large orientation capabilities (taking into account wrench feasibility). The cable exit point positions $A_{i}$ are reconfigured by a maximum of 6 reconfiguration parameters. Four actuators $\left(r_{1}, r_{2}, r_{3}\right.$ and $\left.r_{4}\right)$ are used to drive the cable exit points $A_{i}$ which

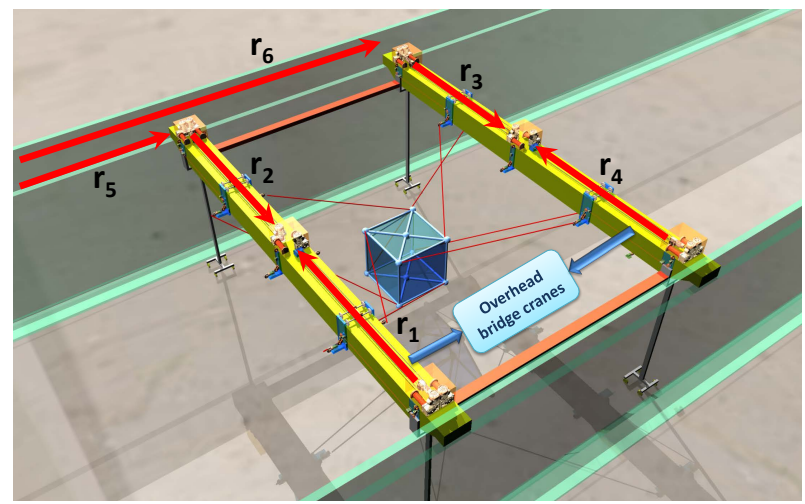

Fig. 2: Solution using large-dimension reconfigurable suspended CDPR to replace conventional cranes 
are moving by pairs along two overhead bridge cranes. The overhead bridge cranes are driven by two actuators $\left(r_{5}, r_{6}\right)$. A systematic procedure was presented to find optimal reconfigurations of the robot where the power consumption of the whole robot system (over a given workspace) is minimized. The reconfiguration of the CDPR is divided into two suboptimization problems. The first one is a vector optimization problem [16] to find the bounds on the reconfiguration parameters where the nonlinear constraints include wrench feasible constraints and non-differentiable constraints (cable interferences or geometric constraints). The general nonlinear optimization problem is then transformed into a singleobjective box-constrained problem that can be solved with readily available gradient-based optimization tools [17]. This approach offers fast solution computations and can be used in both offline or online (real-time) CDPR reconfiguration. However, several issues have to be dealt with. Firstly, the vector optimization problem of finding the bounds on the reconfiguration parameters is complicated. Although a heuristic approach was presented to solve it, the optimal solution greatly depends on specific use cases. Secondly, only one criterion was considered (the minimization of the power consumption of the CDPR). For the application of interest in the present paper, this solution may not be satisfactory enough.

In the present paper, we consider the use of a CDPR to carry workers in an airplane maintenance workshop. The general structure of the CDPR is similar to the one in Fig. 2. The mobile platform is illustrated in Fig. 3. One drawback of this solution is that the CDPR is much more compliant than conventional telescopic platforms (which consist of rigid links). Thus, in order to obtain appropriate CDPR reconfiguration solutions, we take into account two objectives. The first objective is to minimize the power consumption while the second one is to maximize the stiffness of the CDPR. The reconfiguration of the CDPR becomes a multi-objective (or vector) optimization problem with both continuous and non-differentiable nonlinear constraints. The optimal reconfiguration is a Pareto optimal point [18] among a set of possible solutions representing the tradeoff between the two considered criteria.

As the first objective function, we select the sum of cable tensions [15] since this index is directly related to the power consumption of the CDPR. As a second objective function, we introduce a new performance index which is the normalized upper bound on the infinitesimal displacement of the mobile platform as a means to quantify the stiffness of the CDPR. The derivation of this index is based on the study of both the homogeneous CDPR stiffness matrix presented in [19] and the kinematic sensitivity of very large-dimension CDPR presented in [4].

There can be two possible suitable optimization tools to determine the CDPR reconfiguration. If one considers it as a vector optimization problem, methods presented e.g. in [16] or in [20], [21] can be used to compute the Pareto front (the set of Pareto optimal points). However, these methods can deal efficiently with problems that have only

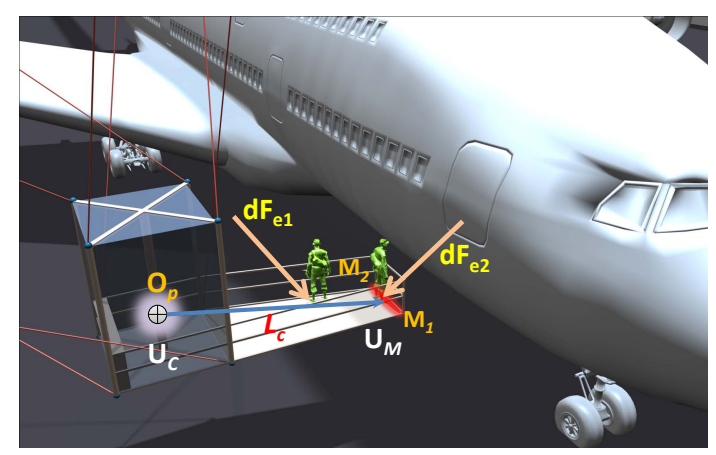

Fig. 3: Mobile platform

linear constraints. Another possibility is to use efficient scalarization methods to govern all the criteria into one objective function and then use available optimization tools to solve this single-objective optimization problem. We choose the global criterion method and bounded objective function method (or $\epsilon$-constrained method) [22] to scalarize multiperformance indices. In order to deal efficiently with the non-differentiable constraints (related to cable collisions), we use a derivative-free optimization tool, e.g., direct search in MATLAB global optimization toolbox [21].

The paper is organized as follows. Section II presents the elastostatic modeling of CDPR and the different collision cases considered in this work. Section III details first the derivation of the two performance indices and their scalarization, and then the procedure to solve the CDPR reconfiguration. Finally, an example illustrating the application of interest is presented in Section IV.

\section{PRELIMINARIES}

\section{A. Inverse kinematics and cable tension distribution}

For large-dimension CDPR handling heavy payloads (could be over 1 ton), hefty steel cables are used because of high safety factors. Cable models with non-negligible mass and elasticity must be taken into account. By using the simplified cable model derived in [8], [23], one can solve the tension distribution problem of a CDPR with hefty steel cables using efficient methods such as the one in [24].

Once the cable tensions are found, the cable unstrained lengths (solution to the inverse kinematic problem) can be computed by using the method presented in [23].

\section{B. Stiffness matrix}

Let us consider the CDPR shown in Figure 4. Assume that each steel cable has unstrained length $L_{0 i}(m)$, self-weight $w(N / m)$, elastic modulus $E(P a)$ and cable cross-section area $A_{0}\left(m^{2}\right) . X=\left(\begin{array}{llllll}x & y & z & \theta_{x} & \theta_{y} & \theta_{z}\end{array}\right)$ denotes the mobile platform pose, $O_{p}=\left(\begin{array}{lll}x & y & z\end{array}\right)$ is the origin of the mobile platform frame, $\theta=\left(\begin{array}{lll}\theta_{x} & \theta_{y} & \theta_{z}\end{array}\right)$ is a vector of Euler angles, $d O_{p}=\left(\begin{array}{lll}d x & d y & d z\end{array}\right)$ is the position displacement vector, $d \Phi=\mathbf{S} \cdot d \theta$ is the rotation displacement vector with $d \theta=$ $\left(\begin{array}{lll}d \theta_{x} & d \theta_{y} & d \theta_{z}\end{array}\right)$ and $d X=\left(d O_{p}, d \Phi\right)$ is the infinitesimal displacement vector of the mobile platform. The term $(\mathbf{u})_{\times}$ indicates the cross product matrix of vector $\mathbf{u}, \mathbf{1}_{3 \times 3}$ is the 


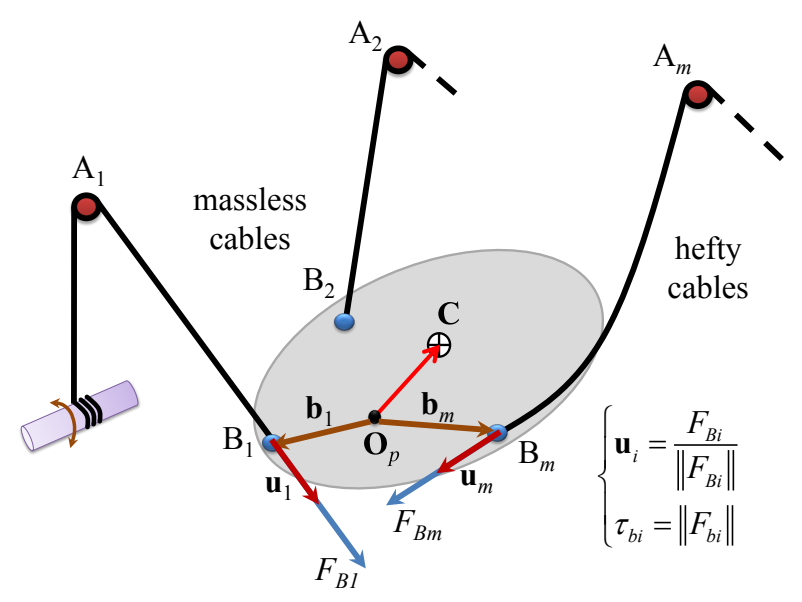

Fig. 4: A general $m$-cable CDPR

identity matrix. The rotation matrix $\mathbf{R}$ and the transformation matrix $\mathbf{S}$ (which maps the time derivative of the vector of ZYX Euler angles to the mobile platform angular velocity vector) are given as follows:

$$
\begin{aligned}
& \mathbf{R}=\left[\begin{array}{ccc}
C_{y} C_{z} & C_{z} S_{y} S_{x}-S_{z} C_{x} & C_{z} S_{y} C_{x}+S_{z} S_{x} \\
C_{y} S_{z} & S_{z} S_{y} S_{x}+C_{z} C_{x} & S_{z} S_{y} C_{x}-C_{z} S_{x} \\
-S_{y} & C_{y} S_{x} & C_{y} C_{x}
\end{array}\right] \\
& \mathbf{S}=\left[\begin{array}{ccc}
C_{y} C_{z} & -S_{z} & 0 \\
C_{y} S_{z} & C_{z} & 0 \\
-S_{y} & 0 & 1
\end{array}\right]
\end{aligned}
$$

with $C_{x}=\cos \left(\theta_{x}\right), S_{x}=\sin \left(\theta_{x}\right), C_{y}=\cos \left(\theta_{y}\right), \ldots$ $F_{B i}=\left(\begin{array}{lll}f_{b i x} & f_{b i y} & f_{b i z}\end{array}\right)$ is the force applied by the mobile platform on the cable $i$ at its end point $B_{i}$. Hence, the resultant wrench applied by the cables on the mobile platform is:

$$
\mathbf{f}_{\mathbf{c}}=\left[\sum_{i=1}^{m}-F_{B i}, \quad \sum_{i=1}^{m}-\left(\mathbf{R} \mathbf{b}_{i} \times F_{B i}\right)\right]^{\mathbf{T}}
$$

Assume that an infinitesimal external wrench $d \mathbf{f}_{\mathbf{e}}$ is applied on the mobile platform. Then, in static equilibrium:

$$
d \mathbf{f}_{\mathbf{e}}=d\left(-\mathbf{f}_{\mathbf{c}}\right)=\left[\begin{array}{c}
\sum_{i=1}^{m} d F_{B i} \\
\sum_{i=1}^{m} d\left(\mathbf{R} \mathbf{b}_{i} \times F_{B i}\right)
\end{array}\right]
$$

which leads to the following relation:

$$
d \mathbf{f}_{\mathbf{e}}=\mathbf{K} \cdot d X-\mathbf{K}_{L} \cdot d L_{0}
$$

where

$$
\mathbf{K}=\left[\begin{array}{ll}
\mathbf{K}_{11} & \mathbf{K}_{12} \\
\mathbf{K}_{21} & \mathbf{K}_{22}
\end{array}\right]
$$

with

$$
\begin{aligned}
\mathbf{K}_{11} & =\sum_{i=1}^{m} \mathbf{K}_{B i} \\
\mathbf{K}_{12} & =\sum_{i=1}^{m} \mathbf{K}_{B i} \cdot\left(\mathbf{R} \mathbf{b}_{i}\right)_{\times} \\
\mathbf{K}_{21} & =\sum_{i=1}^{m}\left(\mathbf{R b}_{i}\right)_{\times} \cdot \mathbf{K}_{B i} \\
\mathbf{K}_{22} & =\sum_{i=1}^{m}\left(F_{B i}\right)_{\times}^{T} \cdot\left(\mathbf{R} \mathbf{b}_{i}\right)_{\times}^{T} \\
& -\sum_{i=1}^{m}\left(\mathbf{R b}_{i}\right)_{\times} \cdot \mathbf{K}_{B i} \cdot\left(\mathbf{R} \mathbf{b}_{i}\right)_{\times}
\end{aligned}
$$

and

$$
\mathbf{K}_{L}=\left[\begin{array}{ccc}
K_{L 1} & \ldots & K_{L m} \\
\left(\mathbf{R b}_{1}\right)_{\times} \cdot K_{L 1} & \ldots & \left(\mathbf{R b}_{m}\right)_{\times} \cdot K_{L m}
\end{array}\right]
$$

where

$$
K_{L i}=\mathbf{K}_{B i} \cdot F_{A i} \cdot\left(\frac{1}{E A_{0}}+\frac{1}{\tau_{a i}}\right) \quad(i=\overline{1, m})
$$

Here, $F_{A i}=\left[\begin{array}{lll}f_{b i x} & f_{b i y} & \left(f_{b i z}-w L_{0 i}\right)\end{array}\right]^{T}$ is the force applied by the cable at point $A_{i}$ (this force points toward $B_{i}$ ). $\mathbf{K}_{B i}$ is the stiffness matrix of the cable $i$ at point $B_{i}$ (can be derived easily by utilizing cable catenary equations [25]), $\tau_{a i}$ is the cable tension at point $A_{i}$.

If during the application of the infinitesimal wrench $d \mathbf{f}_{\mathbf{e}}$ on the mobile platform all the cable unstrained lengths are assumed to be unchanged $\left(d L_{0}=\mathbf{0}\right)$, then we obtain $\mathbf{K}$ as the CDPR stiffness matrix.

In fact, the derivation of the analytical stiffness matrix $\mathbf{K}$ presented here is an improvement of the work done in [26] (which derived the CDPR stiffness matrix based on 2D catenary cable equations and contains errors).

\section{Collision detection}

For cable robots, collisions happen in several cases:

- Interferences between cables and cables

- Interferences between cables and mobile platform

- Interferences between mobile platform and surrounding environment

- Interferences between cables and surrounding environment

In the cases of collisions between the mobile platform and cables with their surrounding environment, one can use AABB trees or OOBB trees method. Indeed, these methods are fast and effective for large objects (triangulation of the mobile platform and obstacles containing a lot of vertices).

For CDPR carrying heavy payloads, the cables become nearly straight, the sagging effect will not really affect the collision detection algorithms. Thus, in the present work, it has been sufficient to consider the collisions between cables as interferences between straight line segments. The 
detection of interferences between the cables and the mobile platform amounts to detecting collisions between line segments and triangles.

Efficient collision detection algorithms can be found in [27].

\section{SOLVING THE CDPR RECONFIGURATION}

\section{A. Performance indices}

1) Sum of cable tensions: Let us consider a CDPR driven by $m$ cables in a configuration defined by vector $\mathbf{r}$ of the reconfiguration parameters. At an equilibrium pose of the mobile platform, by solving the tension distribution problem, we achieve desired cable tensions $\tau_{i}, i=1,2, \ldots, m$. One can compute the sum of the cable tensions at an equilibrium pose:

$$
f_{\tau}(\mathbf{r})=\sum_{i=1}^{m} \tau_{i}(\mathbf{r})
$$

To govern this criteria over a given workspace, we propose to discretized the assigned workspace into $N$ equilibrium poses. In general it is enough to select $N$ extreme points and several internal points of the workspace (e.g. divide the workspace into several boxes and only select the vertices and the center points of these boxes). Then the average sum of cable tensions with respect to a given workspace can be computed as:

$$
f_{\Sigma}(\mathbf{r})=\frac{1}{N} \sum_{k=1}^{N} \sum_{i=1}^{m} \tau_{i k}(\mathbf{r})
$$

where $\tau_{i k}$ is the tension in cable $i$ (at the end point $B_{i}$ ) at the equilibrium pose $k$.

2) Normalized upper bound on the infinitesimal displacement of the mobile platform: Let us consider again the CDPR shown in Fig. 4. In order to quantify the stiffness of the CDPR efficiently, a performance index meaningful for our application of interest is needed. In this work, among a set of reconfiguration solutions, the one which has the "smaller" infinitesimal displacement $d X$ (with respect to any disturbance wrench $d \mathbf{f}_{\mathbf{e}}$ ) is considered to have the higher stiffness. The disturbance wrench can be caused, e.g., by the workers on the platform (see in Fig. 3). From the relation:

$$
\mathbf{K} \cdot d X=d \mathbf{f}_{\mathbf{e}}
$$

one can derive:

$$
\sigma_{\min }=\frac{1}{\left\|\mathbf{K}^{-1}\right\|} \leq \frac{\left\|d \mathbf{f}_{\mathbf{e}}\right\|}{\|d X\|} \leq\|\mathbf{K}\|=\sigma_{\max } \quad(\forall d X \neq \mathbf{0})
$$

where $\sigma_{\min }$ and $\sigma_{\max }$ are the minimum and maximum singular values of the stiffness matrix $\mathbf{K}$, and $\|$.$\| denotes$ the 2-norm of a vector or a matrix.

In this sense, the stiffness of the CDPR can be quantified by the singular values of the stiffness matrix (specifically $\sigma_{\min }$ and $\left.\sigma_{\max }\right)$ regardless of the magnitude or direction of the infinitesimal wrench $d \mathbf{f}_{\mathbf{e}}$. However, the term $\left\|d \mathbf{f}_{\mathbf{e}}\right\|$ and $\|d X\|$ become meaningful only if $d \mathbf{f}_{\mathbf{e}}$ and $d X$ are homogeneous.

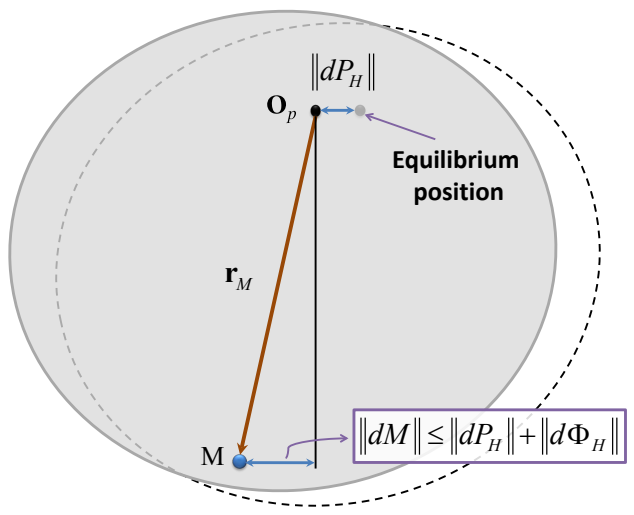

Fig. 5: Displacements of $O_{p}$ and $M$ at the mobile platform of a CDPR

Suppose that from an equilibrium pose, a small disturbance force $d F_{e}$ is applied on the mobile platform at a point $M$. This force creates an infinitesimal wrench at $O_{p}$, $d \mathbf{f}_{\mathbf{e}}=\left(d F_{e}, \quad \mathbf{r}_{M} \times d F_{e}\right) \quad\left(\mathbf{r}_{M}=\overrightarrow{O_{p} M}\right)$. From (9), one can derive:

$$
\mathbf{K}_{H} \cdot d X_{H}=d \mathbf{f}_{\mathbf{e} H}
$$

where

$$
\begin{aligned}
& \mathbf{S}_{H}=\left[\begin{array}{ll}
\mathbf{1}_{3 \times 3} & \mathbf{0}_{3 \times 3} \\
\mathbf{0}_{3 \times 3} & \left\|\mathbf{r}_{M}\right\| \cdot \mathbf{1}_{3 \times 3}
\end{array}\right] \\
& d \mathbf{f}_{\mathbf{e} H}=\left[\begin{array}{c}
d F_{e} \\
\mathbf{u}_{M} \times d F_{e}
\end{array}\right] \\
& d X_{H}=\mathbf{S}_{H} \cdot d X \\
& \mathbf{K}_{H}=\mathbf{S}_{H}^{-1} \cdot \mathbf{K} \cdot \mathbf{S}_{H}^{-1}
\end{aligned}
$$

and $\mathbf{u}_{M}$ is the unit vector $\frac{\mathbf{r}_{M}}{\left\|\mathbf{r}_{M}\right\|}$.

In (11), the terms $d X_{H}$ and $d \mathbf{f}_{\mathbf{e}_{H}}$ are homogeneous (the units are meters and Newtons, respectively). The matrix $\mathbf{S}_{H}$ transforms the stiffness matrix $\mathbf{K}$ into its homogeneous form $\mathbf{K}_{H}$. The characteristic length used in this transformation is $L_{c}=\left\|\mathbf{r}_{M}\right\|$.

Obviously, the choice of the characteristic length $L_{c}$ plays an important role in providing a useful physical meaning for $\mathbf{K}_{H}$. The matrix $\mathbf{K}$ can be considered homogeneous with the trivial characteristic length $L_{c}=\left\|\mathbf{r}_{M}\right\|=1(\mathrm{~m})$. One can then "safely" take the 2-norm of the infinitesimal displacement vector $d X$. However, even if $d X_{H}$ (or $d X$ ) is homogeneous, its components $d P_{H}\left(=d O_{p}\right)$ and $d \Phi_{H}(=$ $\left.\left\|\mathbf{r}_{M}\right\| \cdot d \Phi\right)$ still represent different quantities (position and orientation). Taking the norm of the two terms together in $\left\|d X_{H}\right\|$ and utilize (10) may not be really meaningful. We shall give an interpretation for $d P_{H}$ and $d \Phi_{H}$ as follows.

Let us consider the simple example shown in Figure 5. An infinitesimal wrench $d \mathbf{f}_{\mathbf{e}}$ applied on the mobile platform at point $M$ creates an infinitesimal displacement $d X_{H}=$ $\left(d P_{H}, d \Phi_{H}\right)$. Let us consider the infinitesimal changes in the positions of points $O_{p}$ and $M$ under the action of $d \mathbf{f}_{\mathrm{e}}$. 
One can write:

$$
\begin{gathered}
M=O_{p}+\left(M-O_{p}\right)=O_{p}+\mathbf{r}_{M} \\
\Rightarrow\|d M\|=\left\|d O_{p}+d \Phi \times \mathbf{r}_{M}\right\| \\
\Rightarrow\|d M\| \leq\left\|d O_{p}\right\|+\|d \Phi\| \cdot\left\|\mathbf{r}_{M}\right\| \\
=\left\|d P_{H}\right\|+\left\|d \Phi_{H}\right\|
\end{gathered}
$$

The magnitude of the displacement at $O_{p}$ is $\left\|d P_{H}\right\|$ whereas the magnitude of the displacement at $M$ (with respect to the local mobile platform frame) is bounded by $\left\|d \Phi_{H}\right\|$.

With this interpretation of the homogeneous infinitesimal displacement vector $d X_{H}$, we propose to quantify separately the two terms $d P_{H}$ and $d \Phi_{H}$. From (11), we can write:

$$
\begin{array}{r}
d X_{H}=\mathbf{K}_{H}^{-1} \cdot d \mathbf{f}_{\mathbf{e} H} \\
\Rightarrow\left\{\begin{array}{l}
d P_{H}=\mathbf{C}_{P} \cdot d \mathbf{f}_{\mathbf{e} H} \\
d \Phi_{H}=\mathbf{C}_{\Phi} \cdot d \mathbf{f}_{\mathbf{e} H}
\end{array}\right.
\end{array}
$$

where $\mathbf{K}_{H}^{-1}=\left[\begin{array}{ll}\mathbf{C}_{P}^{T}, & \mathbf{C}_{\Phi}^{T}\end{array}\right]^{T}$.

From (15), we have:

$$
\begin{aligned}
& \frac{\left\|d P_{H}\right\|}{\left\|d \mathbf{f}_{\mathbf{e}_{H}}\right\|} \leq\left\|\mathbf{C}_{P}\right\|=\sigma_{P \max } \\
& \frac{\left\|d \Phi_{H}\right\|}{\| d \mathbf{f}_{\mathbf{e}_{H} \|}} \leq\left\|\mathbf{C}_{\Phi}\right\|=\sigma_{\Phi \max } \\
& \Rightarrow \quad \frac{\|d M\|}{\| d \mathbf{f}_{\mathbf{e}_{H} \|}} \leq \sigma_{M}=\sigma_{P \max }+\sigma_{\Phi \max }
\end{aligned}
$$

where $\sigma_{P \max }$ and $\sigma_{\Phi \max }$ are the maximum singular values of the matrices $\mathbf{C}_{P}$ and $\mathbf{C}_{\Phi}$, respectively.

The term $\sigma_{M}$ is the normalized upper bound on the infinitesimal displacement of the mobile platform and can be used as a means to quantify the stiffness of the CDPR.

To govern this performance index over a given workspace (discretized into $N$ equilibrium poses), we propose to compute the maximum value of $\sigma_{M k}(k=\overline{1, N})$ :

$$
\sigma_{M}(\mathbf{r})=\max _{1 \leq k \leq N}\left\{\sigma_{M k}(\mathbf{r})\right\}
$$

where $\sigma_{M k}$ is the normalized upper bound on the infinitesimal displacement of the mobile platform at the equilibrium pose $k$.

By minimizing $\sigma_{M}(\mathbf{r})$, one can obtain an optimal reconfiguration solution where the potential displacement of the mobile platform at the point $\mathrm{M}$ over a given workspace is minimized.

\section{B. Scalarization of performance indices}

Suppose that $f_{\Sigma}^{*}$ is the optimal value obtained by minimizing $f_{\Sigma}(\mathbf{r})$ in (8) (independently of minimizing $\sigma_{M}(\mathbf{r})$ in (19)) and $\sigma_{M}^{*}$ is the optimal value obtained by minimizing $\sigma_{M}(\mathbf{r})$ in (19).

1) Scalarization using global criteron method: When the two performance indices have the same priority, we propose to use the global criteron method [22]. The reconfiguration can be classically formulated as a singleobjective optimization problem as follows:

$$
\operatorname{minimize} f(\mathbf{r})=\left[\left(1-\delta_{\Sigma}\right)^{s}+\left(1-\delta_{M}\right)^{s}\right]^{1 / s}
$$

subject to:

$$
\begin{aligned}
& \mathbf{r}_{\min } \leq \mathbf{r} \leq \mathbf{r}_{\max } \\
& \mathbf{r} \in \mathbf{C}_{r}
\end{aligned}
$$

where

$$
\delta_{\Sigma}=\frac{f_{\Sigma}(\mathbf{r})}{f_{\Sigma}^{*}}, \quad \delta_{M}=\frac{\sigma_{M}(\mathbf{r})}{\sigma_{M}^{*}}
$$

and $\mathbf{r}_{\min }$ and $\mathbf{r}_{\max }$ are the lower and upper bounds on the reconfiguration parameters. $\mathbf{C}_{r}$ is the set of reconfiguration parameters that satisfy all nonlinear constraints including geometric constraints and wrench feasibility constraints. $s$ is a normalized factor (usually one choose $s=2$ ).

In this way, the tradeoff between the two criteria is managed automatically.

2) Scalarization using bounded objective function method: When the two performance indices have different priorities, we propose to use the bounded objective function method [22] to scalarize the two criteria. One criterion is kept as the objective function while the other criterion is transformed into a nonlinear constraint. For example, assume that there is a strict constraint set upon the power consumption of the CDPR. Then, we can convert $f_{\Sigma}(\mathbf{r})$ into a nonlinear constraint and keep $\sigma_{M}(\mathbf{r})$ as the objective function. The reconfiguration problem in this case becomes:

$$
\text { minimize } \sigma_{M}(\mathbf{r})
$$

subject to:

$$
\begin{aligned}
& \mathbf{r}_{\min } \leq \mathbf{r} \leq \mathbf{r}_{\max } \\
& \mathbf{r} \in \mathbf{C}_{r} \\
& \delta_{\Sigma}(\mathbf{r}) \leq \delta
\end{aligned}
$$

where $\delta$ is a given value.

In this way, the tradeoff between the two criteria is managed by the term $\delta$.

\section{Systematic procedure to solve a reconfiguration problem}

The procedure to find optimal CDPR reconfigurations while optimizing the two objective functions is given in the following steps:

- Step 1: Assign desired CDPR performances including Cartesian workspace and orientation ranges of the mobile platform and bounds on cable tensions (nonlinear constraints $\mathbf{r} \in \mathbf{C}_{r}$ ). Set the limitations of the actuators that drive the cable exit points and the two overhead bridge cranes (linear constraints).

- Step 2: Determine the reconfiguration parameters for the CDPR. The choice of the reconfiguration parameters depends on the use cases.

- Step 3: Determine the characteristic length $L_{c}$ (which transforms the stiffness matrix into its homogeneous form) in order to compute the index $\sigma_{M}$ in (19).

- Step 4a: Find the optimal value $f_{\Sigma}^{*}$ by solving the single-objective optimization problem:

$$
\operatorname{minimize} f_{\Sigma}(\mathbf{r})=\frac{1}{N} \sum_{k=1}^{N} \sum_{i=1}^{m} \tau_{i k}(\mathbf{r})
$$


subject to:

$$
\begin{aligned}
& \mathbf{r}_{\text {min }} \leq \mathbf{r} \leq \mathbf{r}_{\max } \\
& \mathbf{r} \in \mathbf{C}_{r}
\end{aligned}
$$

- Step 4b: Find the optimal value $\sigma_{M}^{*}$ by solving the single-objective optimization problem:

$$
\operatorname{minimize} \sigma_{M}(\mathbf{r})=\max _{1 \leq k \leq N}\left\{\sigma_{M k}(\mathbf{r})\right\}
$$

subject to:

$$
\begin{aligned}
& \mathbf{r}_{\min } \leq \mathbf{r} \leq \mathbf{r}_{\max } \\
& \mathbf{r} \in \mathbf{C}_{r}
\end{aligned}
$$

- Step 5a: If the two performance indices have the same priority, solve the optimization problem (20) to find the Pareto optimal reconfiguration solution for the CDPR.

- Step 5b: If the priorities of the two performance indices are different, solve the optimization problem (22) to find the Pareto optimal reconfiguration solution for the CDPR. The additional nonlinear constraint (e.g., $\delta_{\Sigma}(\mathbf{r}) \leq \delta$ ) should be formulated using the relative ratios given in (21). In case $f_{\Sigma}(\mathbf{r})$ is chosen as the objective function, step $4 \mathrm{a}$ can be removed. In case $\sigma_{M}(\mathbf{r})$ is chosen as the objective function, step $4 \mathrm{~b}$ can be removed.

Note that the nonlinear constraints $\mathbf{r} \in \mathbf{C}_{r}$ can be reformulated into a standard form as follows:

$$
g(\mathbf{r})=0.5-1.0 * h(\mathbf{r}) \leq 0
$$

where

$$
h(\mathbf{r})=\left\{\begin{array}{lll}
1, & \text { if } & \mathbf{r} \in \mathbf{C}_{r} \\
0, & \text { if } & \mathbf{r} \notin \mathbf{C}_{r}
\end{array}\right.
$$

Here, $h(\mathbf{r})$ consists of routines that verify geometric constraints (collision detections) and wrench feasibility constraints.

\section{CASE STUDY}

Let us consider the application of using large-dimension reconfigurable suspended CDPR to replace gantry nacelles in an airplane maintenance workshop, e.g., for the Airbus A380 family. One can use 4 CDPR to cover the workshop divided into four sections across the airplane as shown in Fig. 6. The desired typical trajectory of the mobile platform is to move along the airplane fuselage or along the two wings while carrying 2-3 workers to perform certain maintenance tasks. We will consider the example of one CDPR working in one section of the workshop.

\section{A. Step 1: Assign desired CDPR performances}

In this application, the desired Cartesian workspace consists mainly of the closed area along the airplane fuselage and its wings as shown in Fig. 6. This workspace can be divided into several parts in each of which the mobile platform moves with a constant orientation. We will consider one part of the workspace as shown in Fig. 7. Each bridge crane is of $40 \mathrm{~m}$ in length and $28 \mathrm{~m}$ in height. The distance

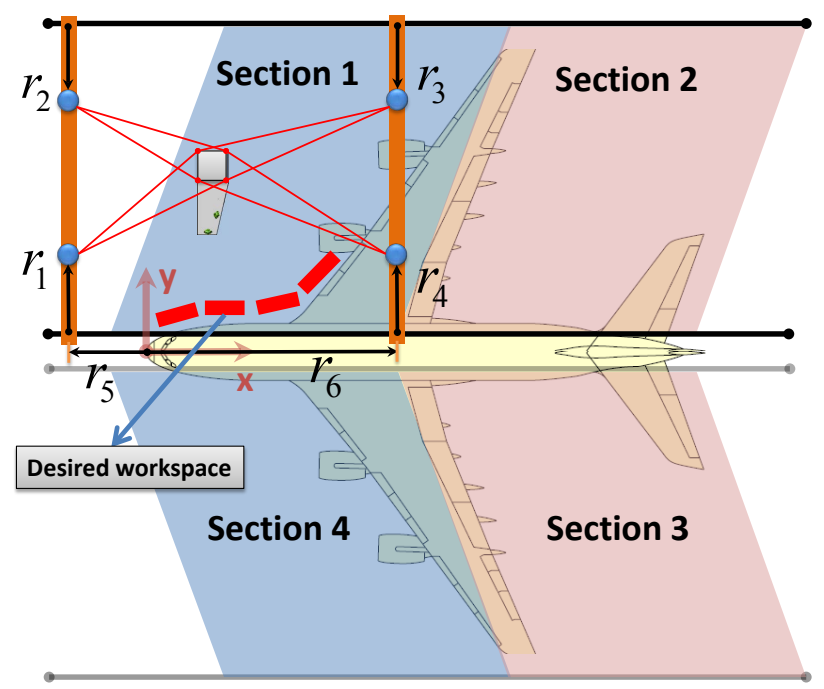

Fig. 6: Using CDPR to replace gantry nacelles in an airplane workshop

between the two cable exit points within a pair mounted on an overhead bridge crane is $2 \mathrm{~m}$. The mobile platform is shown in Fig. 3. Its working area (for the workers) is $4.4 \mathrm{~m}$ long. The mobile platform weighs $3000 \mathrm{~kg}$ and can carry up to 2-3 persons (or $200 \mathrm{~kg}$ in weight). We use steel cable with Young modulus $E=120 e+09 P a$, cross-section area $A_{0}=4.4375 e-05 \mathrm{~m}^{2}$ and self-weight $w=3.3955 \mathrm{~N} / \mathrm{m}$. The desired CDPR performances are given as:

$$
\begin{aligned}
3000 & \leq m_{p} \leq 3200(\mathrm{~kg}) \\
10.0 & \leq x_{p} \leq 20.0(\mathrm{~m}) \\
6.5 & \leq y_{p} \leq 9.0(\mathrm{~m}) \\
1.4 & \leq z_{p} \leq 8.8 \quad(\mathrm{~m}) \\
\theta_{x} & =\theta_{y}=\theta_{z}=0 \quad(\mathrm{deg}) \\
100 & \leq \tau_{i} \leq 3.104 e+04(\mathrm{~N})
\end{aligned}
$$

where $m_{p}$ is the mobile platform weight, $\left(x_{p}, y_{p}, z_{p}\right)$ is the Cartesian position of the origin point $O_{p}$ and $\tau_{i}$ is the tension in cable $i$ at the end point $B_{i}$.

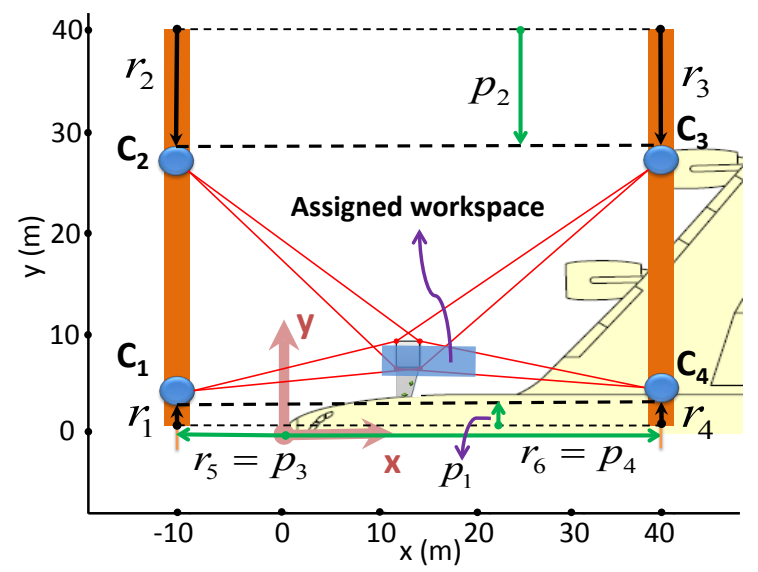

Fig. 7: Reconfiguration parameters 
This Cartesian workspace is discretized into $N=18$ equilibrium poses. The bounds $\left[r_{i \min }, r_{i \max }\right]$ on the parameter $r_{i}$ are given in such a way that the assigned workspace lies within the span of the polygon $C_{1} C_{2} C_{3} C_{4}$ :

$$
\begin{aligned}
0 & \leq r_{1,4} \leq 4.5 \quad(m) \\
0 & \leq r_{2,3} \leq 31(m) \\
-10 & \leq r_{5} \leq 9(m) \\
21 & \leq r_{6} \leq 40 \quad(m)
\end{aligned}
$$

\section{B. Step 2: Determination of the reconfiguration parameters}

There are a maximum of 6 parameters to configure the cable exit points $A_{i}$ of the reconfigurable CDPR considered in this paper (Fig. 2). Here, we consider only one use case where the number of reconfiguration parameters is $n_{r}=4$. Fig. 7 shows the reconfiguration parameters $p_{1}, p_{2}, p_{3}$ and $p_{4}$. Two pairs of cable exit points $C_{1}\left(A_{1}, A_{2}\right)$ and $C_{4}\left(A_{7}, A_{8}\right)$ are reconfigured by $p_{1}\left(r_{1,4}=p_{1}\right)$, while $C_{2}\left(A_{3}, A_{4}\right)$ and $C_{3}\left(A_{5}, A_{6}\right)$ are reconfigured by $p_{2}\left(r_{2,3}=\right.$ $p_{2}$ ). The reconfiguration parameters $p_{3}$ and $p_{4}$ determine the positions of the two overhead bridges $\left(r_{5}=p_{3}, r_{6}=p_{4}\right)$.

Note that after each update of $p_{i}$, a normalization for the parameters $r_{i}$ is needed:

$$
r_{i}=\min \left(\max \left(r_{i}, r_{i \min }\right), r_{i \max }\right) \quad(i=\overline{1,6})
$$

The corresponding bounds on the reconfiguration parameters $p_{i}$ can be determined by using (27).

\section{Step 3: Determination of the characteristic length $L_{c}$}

It can be seen that the homogeneous stiffness matrix $\mathbf{K}_{H}$ of a CDPR presented in Section III-A.b. depends greatly on the choices of:

- The position of the origin $O_{p}$ of the local frame attached to the mobile platform.

- The characteristic length $L_{c}$ (or the choice of point $M$ to be analyzed).

We propose to choose $O_{p}$ as the center of mass $C$ of the mobile platform. In this case, because of the uncertainties on the position of $C$ (due for example to the workers moving on the mobile platform), $C$ falls into a set of possible points $\mathbf{U}_{C}$ (Fig. 3), thus $O_{p}$ (and also the center of mass $C$ ) is chosen as the center point of $\mathbf{U}_{C}$.

To choose $L_{c}$, we propose first to define an area of interest $\mathbf{U}_{M}$ of point $M$ which consists of the most unstable standing positions for the workers. $\mathbf{U}_{M}$ can be chosen as the nearest edge of the mobile platform to the airplane fuselage (the line segment $M_{1} M_{2}$ ). Then, $L_{c}$ is computed as the average distance from $O_{p}$ to the points in $\mathbf{U}_{M}$ as follows:

$$
L_{c}=\int_{0}^{1}\left\|M_{1}+\left(M_{2}-M_{1}\right) \cdot t\right\| d t
$$

Here, $M_{1}$ and $M_{2}$ are expressed in the local mobile platform frame.

By solving (28) we obtain $L_{c}=5.89021 \mathrm{~m}$.
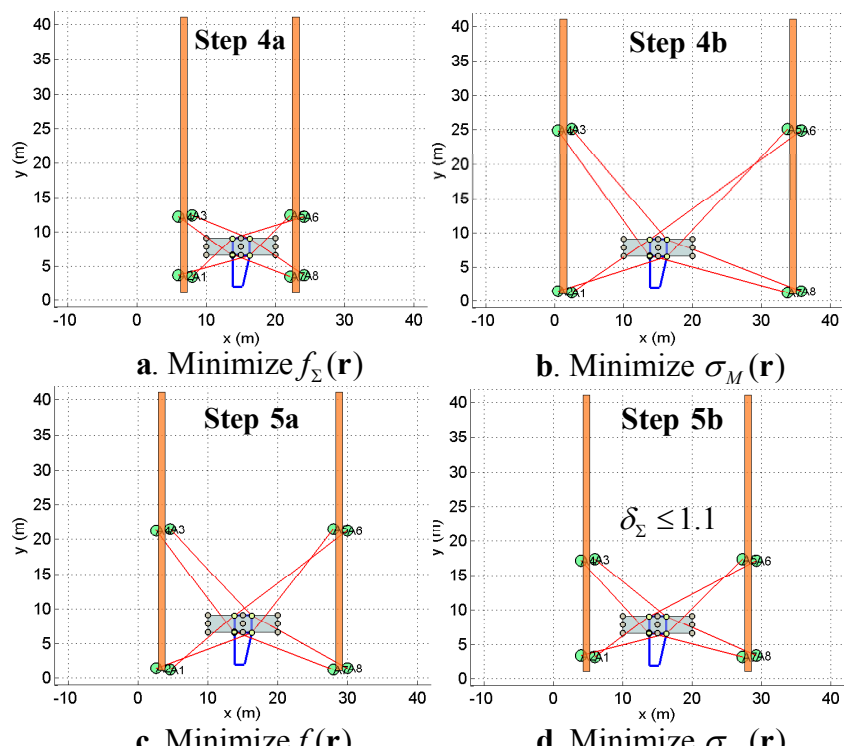

b. Minimize $\sigma_{M}(\mathbf{r})$

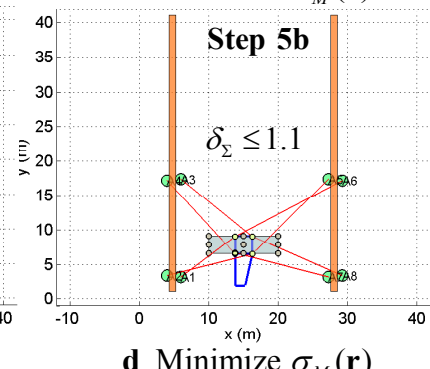

Fig. 8: Reconfiguration solutions in case $n_{r}=4$

\section{Step 4 and 5: Solving the reconfiguration of the CDPR}

We used the pattern search algorithm in MATLAB global optimization toolbox [21] to solve the optimization problems given in steps $4 \mathrm{a}, 4 \mathrm{~b}, 5 \mathrm{a}$ and $5 \mathrm{~b}$ (section III-C). This method handles optimization problems with nonlinear, linear, and bound constraints, and does not require functions to be differentiable or continuous.

The mobile platform weight is $3200 \mathrm{~kg}$ (fully loaded). We only consider static equilibrium poses of the CDPR while neglecting the dynamics of the mobile platform, cables and overhead bridge cranes. In step $5 \mathrm{~b}, \sigma_{M}(\mathbf{r})$ is chosen as the objective function with the additional constraint $\delta_{\Sigma}(\mathbf{r}) \leq \delta=$ 1.1. It means that the power consumption is constrained to be less than $110 \%$ of the optimal value $\left(f_{\Sigma}(\mathbf{r}) \leq 1.1 f_{\Sigma}^{*}\right)$.

In step $4 \mathrm{a}$, we set the initial value $\mathbf{r}_{\text {init }}$ (or $\mathbf{p}_{\text {init }}$ ) of the reconfiguration parameters in the optimization problem as the extreme values $\left(r_{i \max }\right.$ or $\left.r_{i \min }\right)$ where the locations of the cable exit points $A_{i}$ are closest to the assigned workspace [15]. In the following steps $(4 \mathrm{~b}, 5 \mathrm{a}, 5 \mathrm{~b}) \mathbf{r}_{\text {init }}$ is chosen as the optimal configuration found from step $4 \mathrm{a}$.

Fig. 8 shows the optimal reconfiguration solutions in the four steps. It is interesting to note that, in the reconfiguration solution which minimizes the sum of cable tensions, the cables tend to be as vertical as possible (Fig. 8a) [15]. On the contrary, by minimizing the normalized upper bound on the infinitesimal displacement index, we obtain optimal solutions where the cables tend to become horizontal (Fig. 8b). This fact emphasizes the reason why we considered the CDPR reconfiguration as a multi-objective optimization problem.

To illustrate the tradeoff between the two criteria in the four cases, we compute their values and their relative ratios given in (21). The results are shown in Table I (here, $f$ is the objective function defined in (20)). In step 5a, the values of $\delta_{\Sigma}$ and $\delta_{M}$ are quite closed to 1 since we assign equal priorities for the two criteria. Meanwhile, in step 5b, although 
TABLE I: Results obtained by solving the different optimization problems

\begin{tabular}{|c|c|c|c|c|c|c|}
\hline Steps & {$\left[p_{1}, p_{2}, p_{3}, p_{4}\right](m)$} & $f_{\Sigma}(N)$ & $\sigma_{M}(m / N)$ & $f$ & $\delta_{\Sigma}$ & $\delta_{M}$ \\
\hline Step 4a & {$[2.20,28.50,6.75,22.95]$} & $3.6271 e+04$ & $5.8890 e-04$ & 11.4263 & 1.0 & 12.4263 \\
\hline Step 4b & {$[0,15.80,1.25,34.62]$} & $4.5953 e+04$ & $4.7391 e-05$ & 0.2669 & 1.2669 & 1.0 \\
\hline Step 5a & {$[0,19.44,3.38,28.78]$} & $4.1947 e+04$ & $5.1384 e-05$ & 0.1777 & 1.1564 & 1.0843 \\
\hline Step 5b & {$[1.97,23.54,4.77,28.07]$} & $3.9641 e+04$ & $6.8734 e-05$ & 0.4598 & 1.0929 & 1.4503 \\
\hline
\end{tabular}

we obtain a relatively large value of $\delta_{M}$ (because of the constraint set upon $\delta_{\Sigma}$ ), it is still much smaller than the value obtained in step $4 \mathrm{a}$.

\section{CONCLUSIONS}

A reconfigurable suspended CDPR to replace telescopic platforms that carry workers in an airplane workshop has been discussed in this paper. The optimal reconfigurations of this CDPR are found by solving a multi-objective optimization problem with nonlinear constraints. Two performance indices have been introduced. The power consumption of the robot is quantified by the sum of cable tensions, while a new stiffness based index (normalized upper bound on the infinitesimal displacement of the mobile platform) is used to quantify the CDPR stiffness. A systematic procedure to determine the CDPR reconfiguration was presented and illustrated by a case study. It offers the end-users a set of possible solutions where the tradeoffs between the two criteria are explicitly taken into consideration.

Although the use of derivative-free algorithms to solve the CDPR reconfiguration may be time consuming (compared to gradient-based methods), one could effectively handle nondifferentiable nonlinear constraints (geometric constraints), and thus obtain more reliable solutions.

The approach presented in this paper can be used to solve design and planning problems of other (reconfigurable) CDPR.

\section{ACKNOWLEDGMENT}

The research leading to these results has received funding from the European Community's Seventh Framework Programme under grant agreement No. NMP2-SL-2011-285404 (CABLEBOT).

\section{REFERENCES}

[1] J. Albus, R. Bostelman, and N. Dagalakis, "The NIST robocrane," Journal of Robotic Systems, vol. 10, no. 5, pp. 709-724, 1993.

[2] A. B. Alp and S. K. Agrawal, "Cable suspended robots: design, planning and control," Proceedings of IEEE International Conference on Robotics and Automation,ICRA 2002, pp. 4275-4280, 2002.

[3] S.-R. Oh and S. K. Agrawal, "The feasible workspace analysis of a set point control for a cable-suspended robot with input constraints and disturbances," IEEE Transactions on Control Systems Technology, vol. 14, no. 4, pp. 735-742, 2006.

[4] S. Bouchard,C. Gosselin, "Kinematic sensitivity of a very large cabledriven parallel mechanism," ASME International Design Engineering Technical Conferences, 2006.

[5] M. Hassan, A. Khajepour, "Analysis of Large-Workspace CableActuated Manipulator For Warehousing Applications," ASME International Design Engineering Technical Conference, 2009.

[6] B. Duan, Y. Qiu, F. Zhang, B. Zi, "On design and experiment of the feed cable-suspended structure for super antenna," Mechatronics, vol. 19, pp. 503-509, 2009.
[7] N. Riehl, M. Gouttefarde, S. Krut, C. Baradat and F. Pierrot, "Effects of non-negligible cable mass on the static behavior of large workspace cable-driven parallel mechanisms," IEEE International Conference on Robotics and Automation, pp. 2193-2198, 2009.

[8] M. Gouttefarde, J. Collard, N. Riehl, C. Baradat, "Simplified static analysis of large-dimension parallel cable-driven robots," IEEE International Conference on Robotics and Automation, pp. 2299-2305, 2012.

[9] R. Yao, X. Tang, J. Wang, P. Huang, "Dimensional Optimization Design of the Four-Cable-Driven Parallel Manipulator in FAST," IEEE/ASME Transaction on Mechatronics, vol. 15, no. 6, pp. 932941, 2010.

[10] R. Yao, H. Li, X. Zhang, "A Modeling Method of the Cable Driven Parallel Manipulator for FAST," Cable-Driven Parallel Robots, Mechanism and Machine Science, Springer, vol. 12, pp. 423-436, 2013.

[11] J. Lamaury, M. Gouttefarde, "Control of a Large Redundantly Actuated Cable-Suspended Parallel Robots," Proceedings of the IEEE International Conference on Robotics and Automation (ICRA), 2013.

[12] J.-P. Merlet, D. Danney, "A portable, modular parallel wire crane for rescue operations," 2010 IEEE International Conference on Robotics and Automation (ICRA), pp. 2834 - 2839, May 2010.

[13] G. Rosati, D. Zanotto, S. K. Agrawal, "On the Design of Adaptive Cable-Driven Systems," Journal of Mechanisms and Robotics, ASME, vol. 3, May 2011.

[14] X. Zhou, C.P. Tang, and V. Krovi, "Cooperating Mobile Cable Robots: Screw Theoretic Analysis," Lecture Notes in Electrical Engineering, Springer Berlin Heidelberg, vol. 57, pp. 109-123, May 2013.

[15] D. Q. Nguyen, M. Gouttefarde, O. Company and F. Pierrot, "On the Analysis of Large-Dimension Reconfigurable Suspended CableDriven Parallel Robots," IEEE International Conference on Robotics and Automation (ICRA), pp. 5728-5735, 2014.

[16] A. Löhne, Vector Optimization with Infimum and Supremum. Springer, 2011.

[17] S. G. Johnson, "The NLopt nonlinear-optimization package," http://abinitio.mit.edu/nlopt.

[18] Y. Censor, "Pareto Optimality in Multiobjective Problems," Appl. Math. Optimiz., vol. 4, pp. 41-59, 1977.

[19] L. Pascal, Y.-Q. Zheng, Xi.-W. Liu, "Stiffness Analysis of Wire-Driven Parallel Kinematic Manipulators," Proceedings of the 11th World Congress in Mechanism and Machine Science, IFToMM, Tianjin, China, p. April, 2004.

[20] K. Deb, Multi-Objective Optimization using Evolutionary Algorithms. John Wiley \& Sons, Ltd, Chichester, England, 2001.

[21] MATLAB, "Global Optimization Toolbox," http://www.mathworks.com.

[22] S. S. Rao, Engineering Optimization: Theory and Practice. John Wiley \& Sons, 2009.

[23] D. Q. Nguyen, M. Gouttefarde, O. Company and F. Pierrot, "On the simplifications of cable model in static analysis of large-dimension cable-driven parallel robots," IEEE International Conference on Intelligent Robots and Systems (IROS), pp. 928-934, 2013.

[24] J. Lamaury, M. Gouttefarde, "A tension distribution method with improved computational efficiency," Cable-Driven Parallel Robots, Mechanisms and Machine Science, Springer, vol. 12, pp. 71-85, 2013.

[25] H.-T. Thai, S.-E. Kim, "Nonlinear static and dynamic analysis of cable structures," Finite Elements in Analysis and Design, vol. 47, no. 3, pp. 237-246, 2011.

[26] J. Du, H. Bao, X. Duan, C. Cui, "Jacobian analysis of a long-span cable-driven manipulator and its application to forward solution," Mechanism and Machine Theory, vol. 45, pp. 1227-1238, 2010.

[27] C. Ericson, Real-Time Collision Detection. Morgan Kaufmann, 2005. 